\title{
An observational study of Chinese adults with relapsed/refractory Philadelphia-negative acute lymphoblastic leukemia
}

\author{
Jun Ma*,1, Ting $\mathrm{Liu}^{2}$, Jie Jin ${ }^{3}$, Jianda $\mathrm{Hu}^{4}$, Qifa Liư ${ }^{5}$ Jianxiang Wang ${ }^{6}$, Zhixiang Shen ${ }^{7}$, Xin \\ $\mathrm{Du}^{8}$, Bin Jiang ${ }^{9} \&$ Xianhua Meng ${ }^{10,11}$ \\ ${ }^{1}$ Department of Hematology, Harbin Institute of Hematology \& Oncology, Harbin, China \\ ${ }^{2}$ Department of Hematology, West China Hospital of Sichuan University, Chengdu, China \\ ${ }^{3}$ Department of Hematology, The First Affiliated Hospital of Zhejiang University School of Medicine, Hangzhou, Zhejiang Province, \\ China \\ ${ }^{4}$ Fujian Institute of Hematology, Fujian Provincial Key Laboratory on Hematology, Fujian Medical University Union Hospital, Fuzhou, \\ China \\ ${ }^{5}$ Department of Hematology, Southern Medical University Nanfang Hospital, Guangzhou, China \\ ${ }^{6}$ Department of Hematology, Institute of Hematology, Chinese Academy of Medical Sciences, Tianjin, China \\ ${ }^{7}$ Department of Hematology, Shanghai Ruijin Hospital, Shanghai, China \\ ${ }^{8}$ Department of Hematology, Guangdong General Hospital/Guangdong Academy of Medical Sciences, Guangzhou, China \\ ${ }^{9}$ Department of Hematology, Peking University People's Hospital, Beijing, China \\ ${ }^{10}$ Department of Biostatistical Science, Amgen R\&D Asia, Shanghai, China \\ ${ }^{11}$ Boehringer Ingelheim (China) Investment Co., Ltd, Shanghai, China \\ *Author for correspondence: Tel.: +86 4518469 4406; Fax: +86 4518765 0339; majun0322@126.com
}

\section{Summary points}

- The proportion of Chinese patients with relapsed/refractory ALL who achieved complete remission (CR)/CR with partial hematologic recovery after salvage therapy and who received allogeneic hematopoietic stem cell transplant following CR/CR with partial hematologic recovery after salvage therapy is consistent with outcomes reported from US observational studies.

- Data from this observational study of real-world clinical outcomes may provide useful reference material for improving the treatment of this high-risk patient population and for further clinical trials in China.

Aim: Chinese adults with relapsed/refractory Philadelphia chromosome-negative B-cell precursor acute lymphoblastic leukemia (Ph- ALL) have poor outcomes. Patients \& methods: We conducted a nationwide, retrospective, observational study to assess outcomes in this patient population. Results: Of the 270 enrolled patients, $31 \%$ of patients at last salvage achieved complete remission (CR) or CR with partial hematologic recovery (CRh), with median time to CR/CRh of 30 days and median CR/CRh duration of 2.7 months. The CR/CRh rate was more favorable with earlier versus later lines of salvage $(41,24$ and $17 \%$ at first, second and third or later salvages, respectively). Conclusion: This dataset serves as an important reference of real-world outcomes using currently available chemotherapy regimens for high-risk Chinese adults with relapsed/refractory Ph- ALL.

First draft submitted: 7 June 2018; Accepted for publication: 27 June 2018; Published online: 25 July 2018

Keywords: acute lymphoblastic leukemia $\bullet \mathrm{ALL} \bullet$ chemotherapy $\bullet$ China $\bullet$ Philadelphia chromosome-negative B-cell precursor acute lymphoblastic leukemia $\bullet$ relapsed/refractory $\bullet$ retrospective observational study

Relapsed/refractory (R/R) Philadelphia chromosome-negative B-cell precursor acute lymphoblastic leukemia (PhALL) is a rare, aggressive and heterogeneous disease for which there are limited treatment options $[1,2]$. In China, the incidence of ALL in adults is approximately 0.6 per 100,000, and there is notable geographic variation across districts; in Shanghai, one of the largest cities in China, the overall incidence of ALL is 0.81 per 100,000 [3,4].

Future Medicine 
Globally, the treatment of adults with ALL has improved over the past three decades, with complete remission (CR) rates of $85-90 \%$ and long-term overall survival (OS) rates of $30-50 \%$ [5-7]. Despite these advances, a high proportion of patients relapse, with remission rates of $31-44 \%$ after first salvage and $18-25 \%$ after second salvage [8-14]. There is no standard chemotherapy salvage regimen for adults with R/R ALL. Salvage therapy varies by first salvage treatment choice, depth and duration of previous response, overall patient health, regional practice patterns and physician preference $[12,13,15]$. Long-term disease-free survival and cure in $7-24 \%$ of adults with R/R ALL generally occur only after patients have received an allogeneic hematopoietic stem cell transplant (alloHSCT) [8,13,14,16,17].

Outcomes in patients with R/R ALL are even less favorable in China than in western countries [18]. In 2016, a panel of Chinese medical professionals published the Chinese Guidelines for Diagnosis and Treatment of Acute Lymphoblastic Leukemia [19]. This panel reported that the diagnosis and treatment of adult ALL in China required improvement, as most treatment centers lacked a systematic treatment plan and patient outcomes were not satisfactory. Their treatment recommendations for this patient population are induction therapy with regimens including at least vincristine or vinblastine, anthracyclines/anthraquinones and corticosteroids; intensive consolidation therapy following CR; alloHSCT and maintenance therapy; and participation in a clinical trial [19].

We conducted a nationwide, multicenter, retrospective, observational study to determine hematological remission rates and survival outcomes of Chinese adults with $\mathrm{R} / \mathrm{R} \mathrm{Ph}$ - ALL treated with currently available chemotherapy regimens. The primary aim was to collect real-world retrospective data on the clinical treatment of adults with $R / R$ ALL and to provide a reference for the further study of adult R/R ALL treatment in China, with the long-term goal of improving outcomes in this high-risk patient group.

\section{Patients \& methods}

\section{Study design}

This was a retrospective, noninterventional, observational study to collect data on clinical outcomes from Chinese adults who had R/R B-precursor ALL and confirmed Philadelphia chromosome status and who were enrolled from 14 major hematology centers in China (Supplementary Table 1). Eligible patients were at least 15 years old at the time of diagnosis and had at least one evaluable response after salvage therapy. Patients who had achieved $\mathrm{CR}$ on initial induction therapy were required to have an evaluable duration of remission. Patients were excluded if they had central nervous system involvement or isolated extramedullary relapse. This study was conducted in accordance with all applicable regulatory guidelines. The protocol was approved by each study center's institutional review board or independent ethics committee.

\section{Endpoints}

The primary endpoint was the proportion of patients with early relapsed (first remission duration of $\leq 12$ months) or primary refractory $\mathrm{Ph}$ - B-precursor ALL who achieved CR with either full or partial hematologic recovery (CR/CRh) after the last salvage therapy. Secondary endpoints included CR/CRh after the first salvage therapy, and CR with full, partial or incomplete hematologic recovery $(\mathrm{CR} / \mathrm{CRh} / \mathrm{CRi})$, duration of $\mathrm{CR} / \mathrm{CRh}$, duration of $\mathrm{CR} / \mathrm{CRh} / \mathrm{CRi}$ and rate of alloHSCT realization after CR/CRh following first and last salvage therapies. CR was defined as the absence of circulating blasts, absence of extramedullary disease (i.e., no lymphadenopathy, splenomegaly, skin/gum infiltration, testicular mass or central nervous system involvement), trilineage hematopoiesis and less than $5 \%$ blasts by bone marrow assessment, absolute neutrophil counts (ANCs) greater than $1.0 \times 10^{9} / 1$, platelet counts greater than $100 \times 10^{9} / 1$ and no recurrence for at least 4 weeks. CRh criteria were the same as those for CR except for ANC greater than $0.5 \times 10^{9} / 1$ and platelet count greater than $50 \times 10^{9} / \mathrm{l}$. CRi criteria were the same as those for CR except for ANC less than $1.0 \times 10^{9} / 1$ and platelet count less than $100 \times 10^{9} / 1$. Safety data were not collected as part of this study.

\section{Data collection}

Clinical data were collected retrospectively from each patient's original medical records, anonymized and entered into an electronic data collection (EDC) system. Unique record identifiers were applied to the anonymized data to facilitate data quality control procedures. The EDC system was designed to check each variable as well as logical relationships between variables during data collection. Independent study monitors regularly performed quality control checks to ensure consistency between the original medical records and data entered into the EDC system. 


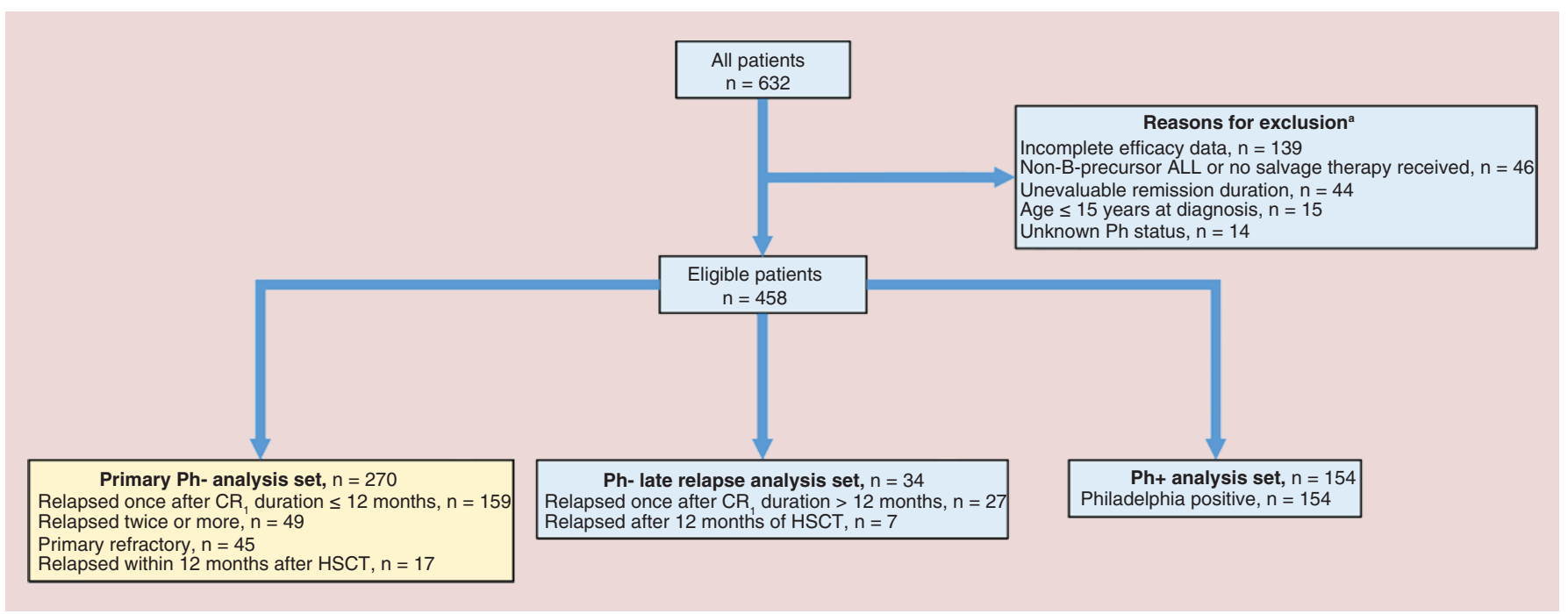

Figure 1. Patient disposition. Data from the primary Ph- patient population are reported.

†Some patients had more than one reason for exclusion.

ALL: Acute lymphoblastic leukemia; $\mathrm{CR}_{1}$ : Complete remission after the first induction therapy; $\mathrm{HSCT}$ : Hematopoietic stem cell transplant; Ph-: Philadelphia chromosome-negative; $\mathrm{Ph}+$ : Philadelphia chromosome-positive.

Investigators applied a rating of data completeness to variables to identify potential misclassification or differences in data provision among sites.

\section{Statistical analyses}

The sample size was based on calculations that would provide reasonable statistical precision and a margin of error of $\pm 9.8 \%$ to $\pm 6.4 \%$ for the estimation of CR/CRh. The primary $\mathrm{Ph}$ - analysis set included patients with $\mathrm{R} / \mathrm{R}$ $\mathrm{Ph}$ - ALL, defined as meeting one of the following criteria: primary refractory, relapsed within 12 months of first $\mathrm{CR}$, relapsed after first or subsequent salvage therapy or relapsed within 12 months of alloHSCT. Continuous variables are summarized using nonmissing sample size, mean, standard deviation, median, interquartile range and range. Categorical variables are summarized using sample size and proportions in each category. Point estimates for efficacy endpoints are accompanied by two-sided $95 \%$ CIs.

The primary analysis was performed on reported data from the last salvage therapy; secondary analyses were based on data from the first salvage therapy. Subgroup analyses assessed the effects of covariates (i.e., line of treatment, refractory status, prior alloHSCT, age, sex, period of initial diagnosis, bone marrow blasts at diagnosis and white blood cell count at diagnosis) with CR/CRh. Univariate and multivariate models used Cox regression analysis to evaluate the relationships between CR/CRh and covariates. Duration of CR/CRh was estimated based on patients with CR/CRh in the primary analysis set and defined from the time when the criteria for CR/CRh were first met until documented hematological relapse or death due to disease progression. Patients who were alive at the end of the study were censored on their last disease assessment date.

\section{Results}

\section{Patient characteristics}

A total of 632 patients with R/R B-precursor ALL were identified from medical records spanning 2005-2014. Among the 458 patients who met all eligibility criteria, 270 had R/R Ph- ALL (17\% were primary refractory, $59 \%$ relapsed within 12 months of first CR, 18\% relapsed twice or more and 6\% relapsed within 12 months after HSCT) and were included in the primary Ph- analysis set (Figure 1). Baseline patient characteristics are presented in Table 1. Approximately half of the patients were male, and the median (range) age was 29 (15-73) years, with the majority younger than 35 years old. 24 (9\%) patients had received prior HSCT (eight autologous HSCT and 16 alloHSCT), and 246 (91\%) patients had not received prior HSCT; the majority of patients had not received prior salvage therapy. At diagnosis, the median (range) bone marrow blast percentage was $84 \%$ (1-99), and the majority of patients ( $73 \%$ ) had $\geq 50 \%$ bone marrow blasts; at last salvage, $39 \%$ of patients had $\geq 50 \%$ bone marrow 
Table 1. Patient characteristics at diagnosis and at last salvage.

Patient characteristics

Ph- patients $(n=270)$

At diagnosis

Sex, $\mathrm{n}(\%)$ :

- Female $125(46)$

- Male 145 (54)

Median (range) age, years $\quad 29(15-73)$

Age group, years, $\mathrm{n}(\%)$ :

$-<35$

-35 to $\leq 55$

$->55 \quad 14$ (5)

Bone marrow blasts, $\mathrm{n}(\%)$ :

$-\leq 5 \%$

$->5 \%$ to $<50 \% \quad 33(12)$

$-\geq 50 \% \quad 197(73)$

- Not available 34 (13)

White blood cell count, $\mathrm{n}(\%)$ :

$-<30,000 / \mu \mathrm{l} 169(63)$

$-\geq 30,000 / \mu \mathrm{l}$

- Not available $26(10)$

At last salvage

Prior HSCT, n (\%):

- Autologous 8 (3)

- Allogeneic 16 (6)

Previous lines of salvage

Prior HSCT, n (\%): 24 (9)

$-0 \quad 18(7)$

$-1 \quad 6(2)$

$-\geq 2 \quad 0$

No prior HSCT, $\mathrm{n}(\%)$ : $\quad 246$ (91)

$-0 \quad 195(72)$

$-1 \quad 45(17)$

$-\geq 2 \quad 6(2)$

Bone marrow blasts, $\mathrm{n}(\%)$ :

$-\leq 5 \% \quad 2(<1)$

$->5 \%$ to $<50 \% \quad 149(55)$

$-\geq 50 \% \quad 105$ (39)

- Not available 14 (5)

HSCT: Hematopoietic stem cell transplant; Ph-: Philadelphia chromosome-negative.

blasts. The last salvage, first salvage and induction regimens in this patient population were diverse, with no specific standard-of-care regimens identified (Supplementary Table 2).

\section{Response}

$31 \%$ (95\% CI: 26-37; $\mathrm{n}=84$ ) of patients achieved CR/CRh at last salvage (primary endpoint), with 21\% (95\% CI: $17-27 ; \mathrm{n}=58)$ achieving CR and $10 \%(95 \% \mathrm{CI}: 6-14 ; \mathrm{n}=26)$ achieving CRh. Overall, $38 \%$ of patients achieved CR/CRh/CRi at last salvage. The proportion of patients who achieved CR/CRh or CR/CRh/CRi at first salvage was 41 and $48 \%$, respectively. Among patients who had CR/CRh, 25\% at first salvage and 29\% at last salvage received an alloHSCT after achieving CR/CRh (Table 2).

The median duration of CR/CRh and CR/CRh/CRi decreased from first to last salvage: from 87 to 82 days for CR/CRh and from 117 to 87 days for CR/CRh/CRi. The median time to CR/CRh was 30 days at first and last salvage (Table 2). 
Table 2. Hematologic response and duration of response.

\begin{tabular}{|c|c|c|}
\hline Patient characteristics & First salvage & Last salvage \\
\hline \multicolumn{3}{|l|}{ CR/CRh: } \\
\hline$-n / N$ & $104 / 270$ & $84 / 270$ \\
\hline - \% (95\% Cl) & $39(33-45)$ & $31(26-37)$ \\
\hline \multicolumn{3}{|l|}{ alloHSCT after CR/CRh: } \\
\hline$-n / N$ & $26 / 104$ & $24 / 84$ \\
\hline - \% (95\% Cl) & $25(17-34)$ & $29(19-39)$ \\
\hline \multicolumn{3}{|l|}{ CR/CRh/CRi: } \\
\hline$-n / N$ & $122 / 270$ & $102 / 270$ \\
\hline - \% (95\% Cl) & $45(39-51)$ & $38(32-44)$ \\
\hline Median duration of $\mathrm{CR} / \mathrm{CRh}$, days $(95 \% \mathrm{Cl})$ : & $87(64-125) ; n=110$ & $82(60-117) ; n=84$ \\
\hline - Relapse, n (\%) & $63(57)$ & $39(46)$ \\
\hline - Death, n (\%) & $8(7)$ & $9(11)$ \\
\hline - Censored, n (\%) & $39(36)$ & $36(43)$ \\
\hline Median time to CR/CRh, days (range) & $30(6-275)$ & $30(6-291)$ \\
\hline Median duration of $\mathrm{CR} / \mathrm{CRh} / \mathrm{CRi}$, days $(95 \% \mathrm{Cl})$ : & $117(78-146) ; n=128$ & $87(67-125) ; n=128$ \\
\hline - Relapse, n (\%) & $60(47)$ & $61(48)$ \\
\hline - Death, n (\%) & $13(10)$ & $13(10)$ \\
\hline - Censored, n (\%) & $55(43)$ & $54(42)$ \\
\hline
\end{tabular}

Subgroup analyses of CR/CRh after first and last salvage therapy showed a significant difference in CR/CRh response and bone marrow blast percentage after first salvage $(\mathrm{p}=0.013) ; 48 \%$ of patients with $>5 \%$ to $<50 \%$ bone marrow blasts achieved CR/CRh (Figure 2). No additional significant interactions between baseline covariates and $\mathrm{CR} / \mathrm{CRh}$ were observed.

Minimal residual disease (MRD) information for patients who had achieved CR/CRh after first and last salvage therapy was available for 12 of 84 and 16 of 84 patients, respectively, among whom $75 \%$ ( 9 of 12 ) and $81 \%$ (13 of 16) achieved an MRD response.

\section{Discussion}

To our knowledge, this is the first retrospective, multicenter, observational study designed to evaluate real-world clinical outcomes in adult patients with $\mathrm{R} / \mathrm{R} \mathrm{Ph}$ - ALL in China. The large patient population from multiple sites across China provided a broad regional representation of patient outcomes and treatment regimens.

The majority of study patients had relapsed within 12 months of first CR (59\%) and had no prior salvage therapy or HSCT (72\%). As expected, the proportion of patients who achieved CR/CRh was higher in first salvage versus last salvage (41 vs $31 \%$, respectively). These outcomes are consistent with those reported from two observational studies of R/R ALL conducted in the USA that reported a CR rate of $31 \%$ after salvage therapy [12] and CR rates of 34 and $18 \%$ after first and second salvage, respectively [10]. In our study, 29\% of patients received alloHSCT following $\mathrm{CR} / \mathrm{CRh}$ after salvage therapy, which is consistent with results from a US observational study in which $25 \%$ of patients received HSCT following a second CR [12]. The outcomes from our study are also comparable to those observed in clinical trials of patients receiving intensive chemotherapy regimens in western countries. For example, the CR rate after salvage was $33-42 \%$ from a pooled analysis of two clinical trials conducted in Germany [8].

A limited number of observational studies have evaluated outcomes in adults with $\mathrm{R} / \mathrm{R} \mathrm{Ph}-\mathrm{ALL}$ in China. For patients who received aclarubicin, cytarabine and GC-SF; high-dose colony-stimulating factor; and hyperfractionated cyclophosphamide, vincristine, doxorubicin and dexamethasone (hyper-CVAD) regimens, CR rates ranged from 45 to $52 \%$ [20]. In a retrospective study in Chinese patients who had relapsed after haploidentical HSCT following chemotherapy plus modified donor lymphocyte infusion, a significantly higher CR rate (64 vs $12.5 \%$ ) and longer median OS (5.57 vs 2.50 months) were reported compared with chemotherapy alone [21]. 


\section{(A) First salvage}

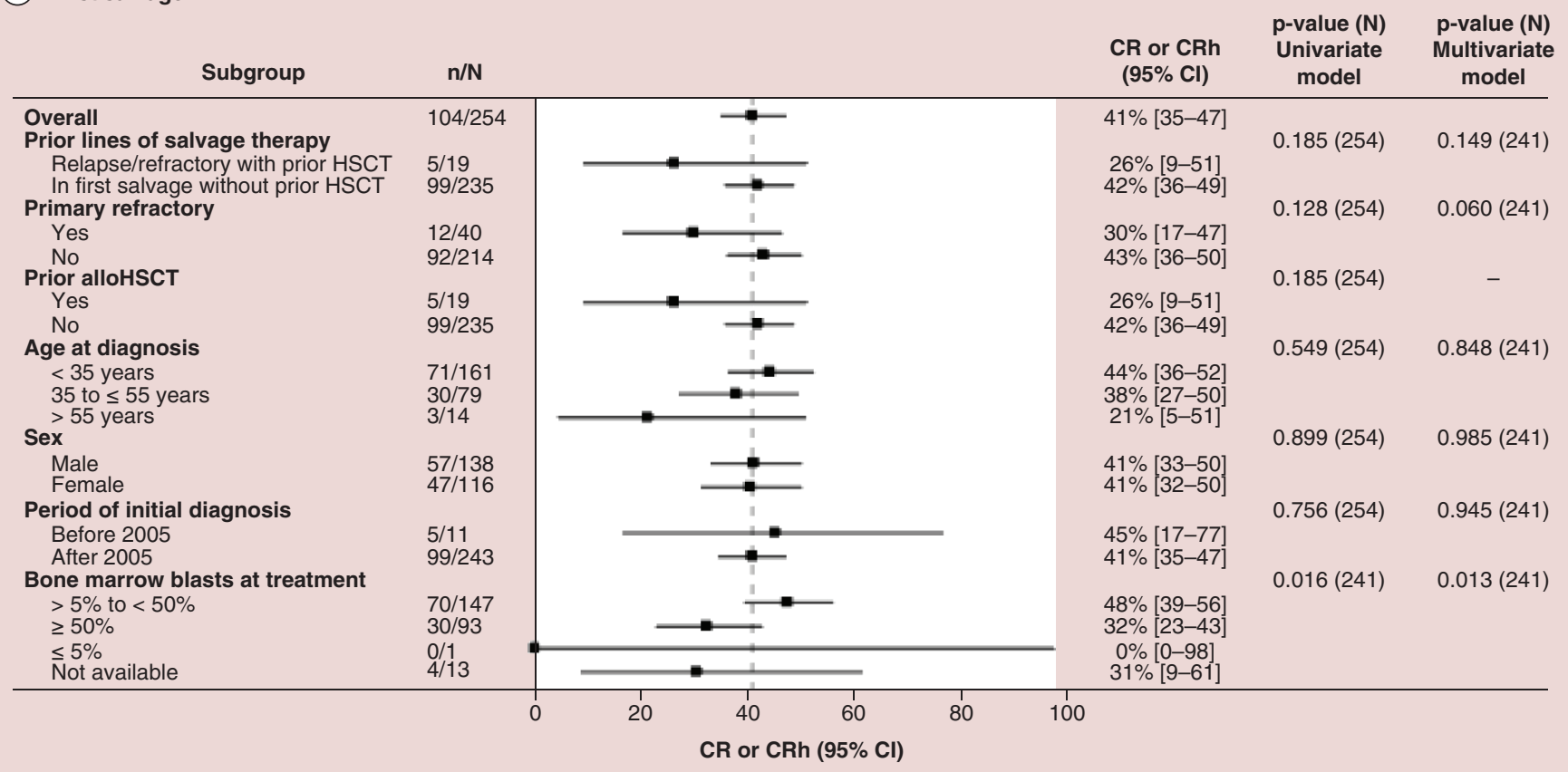

(B) Last salvage

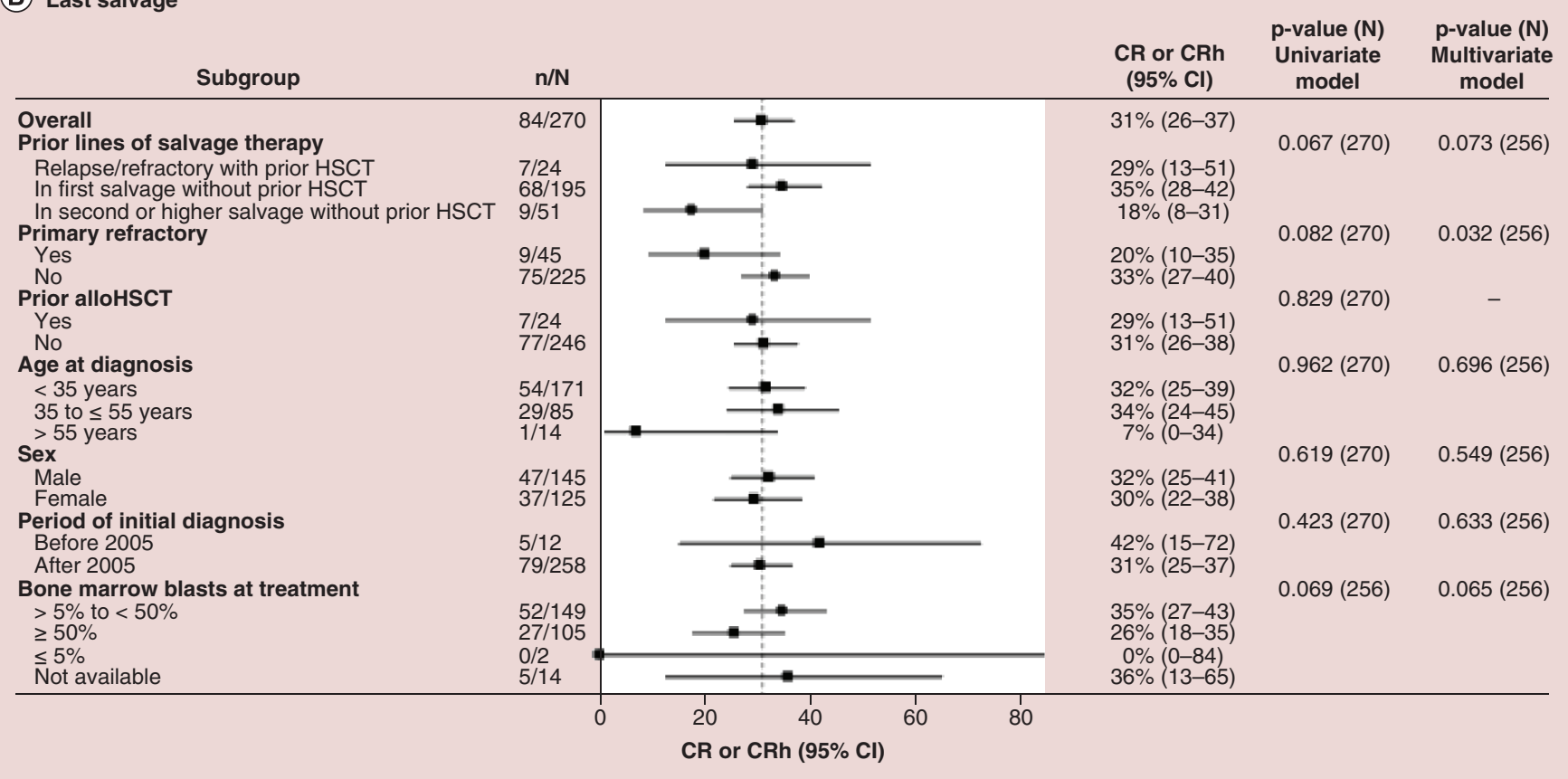

Figure 2. Complete remission/complete remission with partial hematologic recovery after first and last salvage among prespecified patient subgroups. The vertical dashed line represents the point estimate for CR or CRh for the entire patient population.

alloHSCT: Allogeneic hematopoietic stem cell transplant; CR: Complete remission; CRh: CR with partial hematologic recovery; $\mathrm{n} / \mathrm{N}$ : Number of responders/number of total patients within each subgroup. 
The patient population evaluated in our study received a wide variety of intensive combination chemotherapy regimens during induction and salvage therapies, reflecting the heterogeneity of current clinical practices in China with no identified standard of care. Of note, outcomes observed in this Chinese population are consistent with data from western populations treated with similar regimens, a finding that is not totally unexpected, since data were collected from major hematology centers in China. Since our study was completed, immunotherapies such as blinatumomab (an anti-CD3/CD19 bispecific T-cell engager antibody construct approved in a number of countries for R/R Ph- ALL) and the investigational therapies inotuzumab ozogamicin (INO, a CD22 antibodydrug conjugate) and CD19-directed chimeric antigen receptor T cells have improved outcomes in R/R ALL [22-36].

In a randomized Phase III study, blinatumomab monotherapy demonstrated significantly higher CR/CRh/CRi remission rates ( 44 vs $25 \%$; $\mathrm{p}<0.001$ ) and significantly longer median OS (7.7 vs 4.0 months; hazard ratio for death, $0.71 ; 95 \%$ CI: $0.55-0.93 ; \mathrm{p}=0.01)$ versus standard-of-care chemotherapy in adults with high-risk $\mathrm{R} / \mathrm{R}$ B-precursor ALL [25]. Among responders, $76 \%$ of patients who received blinatumomab and $48 \%$ of patients who received chemotherapy also achieved a complete MRD response [25]. In the USA, blinatumomab is included in the National Comprehensive Cancer Network Clinical Practice Guidelines in Oncology for Acute Lymphoblastic Leukemia as the preferred (category 1) treatment option for patients with Ph- ALL.

Among the investigational agents for ALL, INO demonstrated a significantly higher CR versus chemotherapy in patients with relapsed ALL in the first and second salvage treatment settings ( 81 vs $29 \%$; $<<0.001$ ) and a significantly longer median progression-free survival (5.0 vs 1.8 months; $\mathrm{p}<0.001$ ) [9]. CD19-directed chimeric antigen receptor $\mathrm{T}$ cells have demonstrated robust clinical activity in ALL, with CR rates ranging from 70 to $90 \%$ across independent studies, including one in China reporting a CR rate of $87 \%$ [37], and rapid tumor eradication and achievement of MRD-negative CR [28,29,38,39].

Although our study was not designed to collect data on MRD, 12 and $16 \mathrm{CR} / \mathrm{CRh}$ responders after first and last salvage, respectively, had available MRD data, among whom 75\% (9 of 12) and 81\% (13 of 16) achieved an MRD response. As patients with MRD persistence have a higher risk for hematologic relapse, MRD analysis will help to determine the optimal treatment approach for this patient population as more targeted therapies become available in China. For example, blinatumomab treatment of patients with MRD-positive ALL resulted in a complete MRD response rate of $78 \%$, which was associated with longer OS and relapse-free survival compared with those who did not achieve a complete MRD response [40].

A number of baseline factors have been associated with outcomes in patients with ALL, including age and baseline percentage of bone marrow blasts $[8,10,12-14,18,41-45]$. We identified significant correlations between percentage of bone marrow blasts and CR/CRh at first salvage. However, the relatively small size of each subgroup may have prevented the identification of other known predictors of response in this patient population.

Our study has some limitations, which include potential information bias due to differences in reporting among study centers and selection bias due to disproportionate enrollment of patients in certain risk groups. We minimized selection bias by only including patients with confirmed R/R ALL and by performing subgroup analyses. Also, the analysis of endpoints may have been confounded by clinical factors such as time to first relapse. Given that the timeframe for data collection spanned 9 years, treatment patterns may have evolved over that time period. Another key limitation is the study's endpoint. Although OS would have been a more appropriate primary endpoint for our study, CR/CRh was chosen because follow-up information regarding patient survival was unreliable or missing for most of the patients. Finally, the highly specialized institutions selected for our study may not reflect 'real-world' treatments for many patients in China - at the time our study was conducted, there was no national or provincial registry for ALL, and many patients did not receive adequate treatment due to limited medical resources.

In summary, treatment of Chinese patients with R/R ALL is heterogeneous with no identified standard of care, as evidenced by the diverse array of intensive combination chemotherapy regimens utilized during induction and salvage therapy. Although the proportion of Chinese patients with R/R ALL who achieved CR/CRh after salvage therapy and who received alloHSCT following CR/CRh after salvage therapy is consistent with that observed in observational studies of western populations treated with similar regimens, new treatment strategies are required to improve efficacy outcomes in adults with R/R Ph- ALL in China. We believe that data from this observational study of real-world clinical outcomes will provide useful reference material for improving the treatment of this high-risk patient population and for further clinical trials in China. 
Supplementary data

To view the supplementary data that accompany this paper please visit the journal website at: www.futuremedicine.com/doi/suppl/10.2217/ijh-2018-0006

Acknowledgements

The authors thank the patients and the investigators for their participation in this study.

Financial \& competing interests disclosure

This study was sponsored by Amgen China R\&D Center, Shanghai, China. J Ma, T Liu, J Jin, J Hu, Q Liu, J Wang, Z Shen, X Du and $B$ Jiang declare no conflict of interest. X Meng was employed by Amgen Inc. and owned stock in Amgen Inc. at the time of this study. The authors have no other relevant affiliations or financial involvement with any organization or entity with a financial interest in or financial conflict with the subject matter or materials discussed in the manuscript apart from those disclosed.

KJ Boorer (KB Scientific Communications LLC), whose work was funded by Amgen Inc., and JY Ahn (Amgen Inc.) provided medical writing and editorial support.

\section{Open access}

This work is licensed under the Attribution-NonCommercial-NoDerivatives 4.0 Unported License. To view a copy of this license, visit http://creativecommons.org/licenses/by-nc-nd/4.0/

ClinicalTrials.gov (identifier: NCT03123887).

\section{References}

Papers of special note have been highlighted as: $\bullet$ of interest; $\bullet \bullet$ of considerable interest

1. Pui CH, Robison LL, Look AT. Acute lymphoblastic leukaemia. Lancet 371(9617), 1030-1043 (2008).

2. Bassan R, Gatta G, Tondini C, Willemze R. Adult acute lymphoblastic leukaemia. Crit. Rev. Oncol. Hematol. 50(3), $223-261$ (2004).

3. Katz AJ, Chia VM, Schoonen WM, Kelsh MA. Acute lymphoblastic leukemia: an assessment of international incidence, survival, and disease burden. Cancer Causes Control 26(11), 1627-1642 (2015).

4. Ni X, Shen ZX, Chen FY et al. Trend in the incidence and geographic variations of acute lymphoblastic leukemia in Shanghai, China from 2002 to 2006. Chin. Med. J. (Engl.) 124(16), 2406-2410 (2011).

5. Bassan R, Hoelzer D. Modern therapy of acute lymphoblastic leukemia. J. Clin. Oncol. 29(5), 532-543 (2011).

6. Kantarjian H, Thomas D, O'brien S et al. Long-term follow-up results of hyperfractionated cyclophosphamide, vincristine, doxorubicin, and dexamethasone (hyper-CVAD), a dose-intensive regimen, in adult acute lymphocytic leukemia. Cancer 101(12), 2788-2801 (2004).

7. Gökbuget N, Hoelzer D, Arnold R et al. Treatment of adult ALL according to protocols of the German multicenter study group for adult ALL (GMALL). Hematol. Oncol. Clin. North Am. 14(6), 1307-1325 (2000).

8. Gökbuget N, Stanze D, Beck J et al. Outcome of relapsed adult lymphoblastic leukemia depends on response to salvage chemotherapy, prognostic factors, and performance of stem cell transplantation. Blood 120(10), 2032-2041 (2012).

9. Kantarjian HM, Deangelo DJ, Stelljes $\mathrm{M}$ et al. Inotuzumab ozogamicin versus standard care for acute lymphoblastic leukemia. $N$. Engl. J. Med. 375(8), 740-753 (2016).

- Data demonstrating rate of complete remission was higher with inotuzumab ozogamicin than with standard therapy in adults with acute lymphoblastic leukemia (ALL); veno-occlusive liver disease was a major adverse event associated with inotuzumab ozogamicin.

10. O'Brien S, Thomas D, Ravandi F et al. Outcome of adults with acute lymphocytic leukemia after second salvage therapy. Cancer 113(11), 3186-3191 (2008).

11. Tavernier E, Boiron JM, Huguet $\mathrm{F}$ et al. Outcome of treatment after first relapse in adults with acute lymphoblastic leukemia initially treated by the LALA-94 trial. Leukemia 21(9), 1907-1914 (2007).

12. Thomas DA, Kantarjian H, Smith TL et al. Primary refractory and relapsed adult acute lymphoblastic leukemia: characteristics, treatment results, and prognosis with salvage therapy. Cancer 86(7), 1216-1230 (1999).

13. Fielding AK, Richards SM, Chopra R et al. Outcome of 609 adults after relapse of acute lymphoblastic leukemia (ALL); an MRC UKALL12/ECOG 2993 study. Blood 109(3), 944-950 (2007).

14. Oriol A, Vives S, Hernández-Rivas JM et al. Outcome after relapse of acute lymphoblastic leukemia in adult patients included in four consecutive risk-adapted trials by the PETHEMA Study Group. Haematologica 95(4), 589-596 (2010).

15. Lee KJ, Chow V, Weissman A, Tulpule S, Aldoss I, Akhtari M. Clinical use of blinatumomab for B-cell acute lymphoblastic leukemia in adults. Ther. Clin. Risk Manag. 12, 1301-1310 (2016).

16. Duval M, Klein JP, He W et al. Hematopoietic stem-cell transplantation for acute leukemia in relapse or primary induction failure. $J$. Clin. Oncol. 28(23), 3730-3738 (2010). 
17. Frey NV, Luger SM. How I treat adults with relapsed or refractory Philadelphia chromosome-negative acute lymphoblastic leukemia. Blood 126(5), 589-596 (2015).

18. Mi JQ, Wang X, Yao Y et al. Newly diagnosed acute lymphoblastic leukemia in China (II): prognosis related to genetic abnormalities in a series of 1091 cases. Leukemia 26(7), 1507-1516 (2012).

19. Hematology Oncology Committee Ca-CaLLG, Chinese Society of Hematology, Chinese Medical Association. Chinese guidelines for diagnosis and treatment of acute lymphoblastic leukemia (2016). Chin. J. Hematol. 37(10), 837-845 (2016).

•• Described treatment recommendations for adults with ALL, including induction regimens with vincristine or vinblastine, anthracyclines/anthraquinones and corticosteroids; intensive consolidation therapy following complete remission; allogeneic hematopoietic stem cell transplant and maintenance therapy; and participation in a clinical trial.

20. Liu L, Jiao W, Zhang Y, Qu Q, Li X, Wu D. Efficacy of low-dose cytarabine and aclarubicin in combination with granulocyte colony-stimulating factor (CAG regimen) compared to Hyper-CVAD regimen as salvage chemotherapy in relapsed/refractory Philadelphia chromosome-negative acute lymphoblastic leukemia. Leuk. Res. 39(3), 323-328 (2015).

21. Yan CH, Wang JZ, Liu DH et al. Chemotherapy followed by modified donor lymphocyte infusion as a treatment for relapsed acute leukemia after haploidentical hematopoietic stem cell transplantation without in vitro T-cell depletion: superior outcomes compared with chemotherapy alone and an analysis of prognostic factors. Eur. J. Haematol. 91(4), 304-314 (2013).

22. Issa G, Kantarjian H, Ravandi F et al. Updated results from the Phase II study of hyper-CVAD in combination with ofatumumab as frontline therapy for adults with CD20-positive (CD20+) acute lymphoblastic leukemia (ALL). J. Clin. Oncol. 34, Abstract 7042 (2016).

23. Hoelzer D, Gökbuget N. Chemoimmunotherapy in acute lymphoblastic leukemia. Blood Rev. 26(1), 25-32 (2012).

24. Jabbour E, Kantarjian H. Immunotherapy in adult acute lymphoblastic leukemia: the role of monoclonal antibodies. Blood Adv. 1(3), 260-264 (2016).

25. Kantarjian H, Stein A, Gokbuget N et al. Blinatumomab versus chemotherapy for advanced acute lymphoblastic leukemia. N. Engl. J. Med. 376(9), 836-847 (2017).

- Data demonstrating treatment with blinatumomab resulted in significantly longer overall survival than chemotherapy among adults with relapsed/refractory B-cell precursor ALL.

26. Kantarjian H, Thomas D, Jorgensen J et al. Inotuzumab ozogamicin, an anti-CD22-calecheamicin conjugate, for refractory and relapsed acute lymphocytic leukaemia: a Phase II study. Lancet Oncol. 13(4), 403-411 (2012).

27. Kantarjian H, Thomas D, Jorgensen J et al. Results of inotuzumab ozogamicin, a CD22 monoclonal antibody, in refractory and relapsed acute lymphocytic leukemia. Cancer 119(15), 2728-2736 (2013).

28. Lee DW, Kochenderfer JN, Stetler-Stevenson M et al. T cells expressing CD19 chimeric antigen receptors for acute lymphoblastic leukaemia in children and young adults: a Phase I dose-escalation trial. Lancet 385(9967), 517-528 (2015).

29. Maude SL, Frey N, Shaw PA et al. Chimeric antigen receptor T cells for sustained remissions in leukemia. N. Engl. J. Med. 371(16), 1507-1517 (2014).

30. Maury S, Chevret S, Thomas X et al. Rituximab in B-lineage adult acute lymphoblastic leukemia. N. Engl. J. Med. 375(11), 1044-1053 (2016).

31. Thomas DA, O’brien S, Faderl S et al. Chemoimmunotherapy with a modified hyper-CVAD and rituximab regimen improves outcome in de novo Philadelphia chromosome-negative precursor B-lineage acute lymphoblastic leukemia. J. Clin. Oncol. 28(24), 3880-3889 (2010).

32. Thomas X. Inotuzumab ozogamicin in the treatment of B-cell acute lymphoblastic leukemia. Expert Opin. Investig. Drugs 21(6), 871-878 (2012).

33. Topp MS, Gokbuget N, Stein AS et al. Safety and activity of blinatumomab for adult patients with relapsed or refractory B-precursor acute lymphoblastic leukaemia: a multicentre, single-arm, Phase II study. Lancet Oncol. 16(1), 57-66 (2015).

34. Yilmaz M, Richard S, Jabbour E. The clinical potential of inotuzumab ozogamicin in relapsed and refractory acute lymphocytic leukemia. Ther. Adv. Hematol. 6(5), 253-261 (2015).

35. Zugmaier G, Gökbuget N, Klinger M et al. Long-term survival and T-cell kinetics in relapsed/refractory ALL patients who achieved MRD response after blinatumomab treatment. Blood 126(24), 2578-2584 (2015).

36. Martinelli G, Boissel N, Chevallier P et al. Complete hematologic and molecular response in adult patients with relapsed/refractory philadelphia chromosome-positive B-precursor acute lymphoblastic leukemia following treatment with blinatumomab: results from a Phase II, single-arm, multicenter study. J. Clin. Oncol. 35(16), 1795-1802 (2017).

37. Xiao L, Huang H, Huang X et al. Efficacy of anti-CD19 chimeric antigen receptor modified T(CAR-T) cell therapy in Chinese patients with relapsed/refractory acute lymphocytic leukemia in a multicenter trial. J. Clin. Oncol. 35, Abstract 7028 (2017).

- Data demonstrating the successful treatment of relapsed/refractory ALL with anti-CD19 chimeric antigen receptor T cells in China.

38. Davila ML, Riviere I, Wang X et al. Efficacy and toxicity management of $19-28 \mathrm{z}$ CAR T cell therapy in B cell acute lymphoblastic leukemia. Sci. Transl. Med. 6(224), 224 ra225 (2014). 
39. Brentjens RJ, Davila ML, Riviere I et al. CD19-targeted T cells rapidly induce molecular remissions in adults with chemotherapy-refractory acute lymphoblastic leukemia. Sci. Transl. Med. 5(177), 177ra138 (2013).

40. Gokbuget N, Dombret $\mathrm{H}$, Bonifacio $\mathrm{M}$ et al. Blinatumomab for minimal residual disease in adults with B-cell precursor acute lymphoblastic leukemia. Blood 131(14), 1522-1531 (2018).

- Data demonstrating a complete minimal residual disease (MRD) response in a population of patients with MRD-positive B-cell precursor ALL following treatment with blinatumomab, resulting in longer RFS and overall survival compared with MRD nonresponders.

41. Bao L, Gross SA, Ryder J et al. Adult precursor B lymphoblastic leukemia in Shanghai, China: characterization of phenotype, cytogenetics and outcome for 137 consecutive cases. Int. J. Hematol. 89(4), 431-437 (2009).

42. Marks DI, Moorman AV, Chilton L et al. The clinical characteristics, therapy and outcome of 85 adults with acute lymphoblastic leukemia and $\mathrm{t}(4 ; 11)$ (q21;q23)/MLL-AFF1 prospectively treated in the UKALLXII/ECOG2993 trial. Haematologica 98(6), 945-952 (2013).

43. Matsumura T, Kami M, Yamaguchi T et al. Allogeneic cord blood transplantation for adult acute lymphoblastic leukemia: retrospective survey involving 256 patients in Japan. Leukemia 26(7), 1482-1486 (2012).

44. Shaikh MU, Ali N, Adil SN, Khurshid M. Outcome of adult patients with acute lymphoblastic leukaemia receiving the MRC UKALL XII protocol: a tertiary care centre experience. Singapore Med. J. 52(5), 370-374 (2011).

45. Annino L, Vegna ML, Camera A et al. Treatment of adult acute lymphoblastic leukemia (ALL): long-term follow-up of the GIMEMA ALL 0288 randomized study. Blood 99(3), 863-871 (2002). 